\title{
Direct observation of optically induced transient structures in graphite using ultrafast electron crystallography
}

\author{
Ramani K. Raman, Yoshie Murooka, Chong-Yu Ruan* Teng Yang, Savas Berber, and David Tománek \\ Physics and Astronomy Department, Michigan State University, East Lansing, Michigan 48824-2320
}

\begin{abstract}
We use ultrafast electron crystallography to study structural changes induced in graphite by a femtosecond laser pulse. At moderate fluences of $\leq 21 \mathrm{~mJ} / \mathrm{cm}^{2}$, lattice vibrations are observed to thermalize on a time scale of $\approx 8 \mathrm{ps}$. At higher fluences approaching the damage threshold, lattice vibration amplitudes saturate. Following a marked initial contraction, graphite is driven nonthermally into a transient state with $s p^{3}$-like character, forming interlayer bonds. Using $a b$ initio density functional calculations, we trace the governing mechanism back to electronic structure changes following the photo-excitation.
\end{abstract}

PACS numbers: 82.53.Mj, 81.05.Uw, 61.14.-x, 65.40.De

There is growing interest in displacing atoms in materials by photo-excitations [1]. Observations of transient structures thus formed offer a glimpse into the transformation pathways between different structures. Carbon, with its propensity to form a wide range of bonding networks $\left(s p, s p^{2}, s p^{3}\right)$ is ideal to study the dynamics of bond formation and rupture. Of particular interest is the conversion of graphite to diamond [2, 3], which is believed to involve the rhombohedral phase of graphite as intermediate state [4, 5]. Whereas pioneering ultrafast optical studies of graphite have provided evidence for photo-induced melting [6, 7] and generation of coherent phonons [8, 9] by observing changes in the electronic properties, direct determination of lattice structural dynamics from optical data proves difficult, especially in the far-from-equilibrium regime. X-ray diffraction has been successful in observing nonthermal structural changes in semiconductors [10], relying primarily on the integrated Bragg intensities. However, direct observation of atomic motion in nanostructures with low atomic number, such as carbon, has not yet been achieved. Electron diffraction, with its five orders of magnitude enhanced scattering cross-section and advanced schemes achieving femtosecond temporal resolution [11, 12, 13, 14], offers a new window into the realm of photo-excited structural dynamics, with sensitivity down to $\lesssim 1 \mathrm{~nm}[11,14,15]$.

Here, we report the first direct determination of structural changes induced in graphite by a femtosecond laser pulse. At moderate fluences of $\leq 21 \mathrm{~mJ} / \mathrm{cm}^{2}$, after a short thermalization period of $\approx 8 \mathrm{ps}$, we can attribute a temperature value to the graphitic layers and find the interlayer vibration amplitudes to match those reported in X-ray and neutron scattering studies [16]. At higher fluence values approaching the damage threshold, we observe lattice vibration amplitudes to saturate. Following a marked initial contraction of the interlayer spacing by $\leq 6 \%$, graphite is driven nonthermally into a transient state with $s p^{3}$-like character, forming $1.9 \AA$ long interlayer bonds. Using $a b$ initio density functional theory (DFT) calculations, we trace the structural changes back

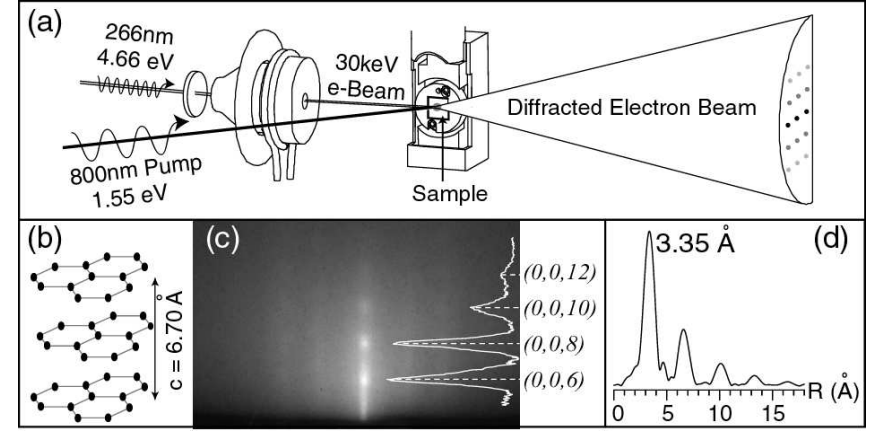

FIG. 1: Ultrafast Electron Crystallography (UEC) of highly oriented pyrolytic graphite. (a) The UEC pump-probe setup. (b) The layered structure of graphite. (c) Ground state diffraction pattern of graphite. (d) Ground state layer density distribution function (LDF), obtained via a Fourier transform of the central streak pattern in (c).

to a nonthermal heating of the electron gas, followed by a photo-induced charge separation causing a compressive Coulomb stress.

The experimental setup, shown in Fig. 1(a), has been described in detail elsewhere [14]. Freshly cleaved samples of highly oriented pyrolytic graphite (HOPG) were placed inside an ultrahigh vacuum chamber through a load lock system at room temperature. A mode-locked Ti-Sapphire laser ( $p$-polarized, $45 \mathrm{fs}, 800 \mathrm{~nm}, 1 \mathrm{kHz}$ ) generated the pump pulse, which was marginally focused $(\approx 600 \mu \mathrm{m}$ diameter $)$ onto the sample at $\approx 45^{\circ}$ relative to the HOPG c-axis. The range of the laser fluence $0.5<F<90 \mathrm{~mJ} / \mathrm{cm}^{2}$ was below the in situ determined optical damage threshold of $\approx 120 \mathrm{~mJ} / \mathrm{cm}^{2}$.

A photo-generated $30 \mathrm{keV}$ electron beam (wavelength $\lambda_{e}=0.069 \AA$ ), with diameter demagnified to $\leq 5 \mu \mathrm{m}$, was directed onto the sample at grazing incidence of $2.4^{\circ}$ to serve as the probe. A translational optical delay stage was used to vary the relative delay between the arrival of pump and probe pulses at the sample, to observe the changes induced by laser irradiation. The electron pulse sampled the top $3-5$ layers of the graphite structure, 
depicted in Fig. 1(b), and produced oriented molecular diffraction patterns in the central streak region, shown in Fig. 1(c). The layer density distribution function (LDF), shown in Fig. 1(d), was obtained from the Fourier analysis of the interference pattern. The primary peak at $3.35 \AA$ and less pronounced peaks at $6.7 \AA$ and $\approx 10 \AA$ were found to be in good agreement with the layered structure of bulk graphite [16]. The decay of higher order LDF peaks suggest a probing depth of $\approx 1 \mathrm{~nm}$.

To study the photo-induced structure dynamics, we first examine the near-equilibrium regime at low fluences of $0.5 \mathrm{~mJ} / \mathrm{cm}^{2} \leq F \leq 21 \mathrm{~mJ} / \mathrm{cm}^{2}$. Random atomic displacements diminish the intensity of the diffraction spectra by a Debye-Waller factor $e^{-2 M}$, where $M=-s^{2} \bar{u}^{2} / 4$, $\bar{u}^{2}$ is the mean-square atomic displacement perpendicular to the reflecting planes, and $s=\left(4 \pi / \lambda_{e}\right) \sin (\theta / 2)$ is the momentum transfer associated with the maxima located at the scattering angle $\theta$. Thus, the change in the meansquare atomic displacement $\Delta \bar{u}^{2}$, measured relative to the unperturbed state at negative times, determines the rise in lattice temperature and can be calculated from the diffraction intensities as $\ln \left(I_{s}(t) / I_{s}(t<0)\right)=-s^{2} \Delta \bar{u}^{2} / 4$. In Ultrafast Electron Crystallography (UEC), we monitor the integrated intensity of the $(0,0,6),(0,0,8)$ and the $(0,0,10)$ maxima arising from the interference between the graphitic layers, shown in Fig. 2(a). The intensity of all 3 maxima drops within a timescale of $8 \pm 1 \mathrm{ps,} \mathrm{indica-}$ tive of increased thermal motion of the graphitic planes. As we probe reflections from the basal planes, the observed intensity drops mainly due to the increased outof-plane displacement of the atoms. Recent optical studies [8, 9] have associated near-infrared optical excitations in graphite with a femtosecond generation of coherent phonons with $E_{2 g}$ symmetry (interlayer shearing mode). Hence, this 8 ps timescale of interlayer thermal excitation is a direct measure of the phonon-phonon interactions in HOPG, and is in good agreement with reports of hot phonon relaxation times in graphite [17].

Quantitative analysis of the intensity drops in Fig. 2(a) indicates that the mean square atomic displacement $\Delta \bar{u}^{2}$ increases linearly with the applied fluence in the nearequilibrium regime, as seen in Fig. 2(b). Comparing our data to a theoretical model of temperature dependent $\Delta \bar{u}^{2}$, benchmarked with X-Ray and neutron scattering data [16], we estimate a temperature rise of $950 \mathrm{~K}$ at $F=$ $21 \mathrm{~mJ} / \mathrm{cm}^{2}$ in Fig. 2(b). This is also in good agreement with temperature extracted from optical studies using heat capacity and absorbance [7].

As we enter the far-from-equilibrium regime by increasing the excitation fluence beyond $21 \mathrm{~mJ} / \mathrm{cm}^{2}$, the amplitude of atomic vibrations saturates and no longer increases with increasing fluence, as seen in Fig. 2(b). This is rather surprising, for one would expect a monotonic increase in the thermal motion as graphite approaches its melting threshold at $130 \mathrm{~mJ} / \mathrm{cm}^{2}$ [7]. Furthermore, the $(0,0,6)$ maximum is found to shift by almost $0.34 \AA^{-1}$ (a)
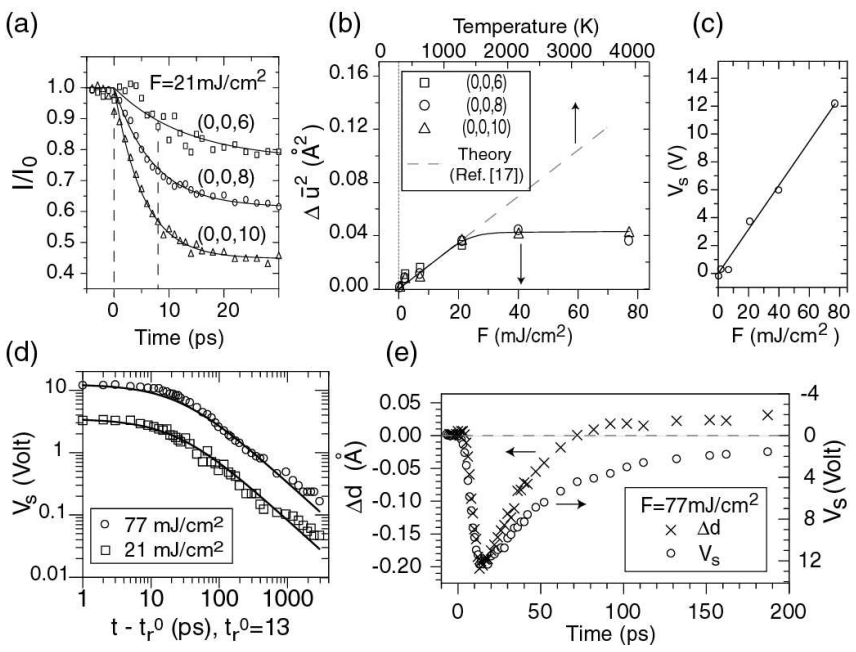

FIG. 2: (a) Decay of the diffraction intensity. (b) Change in mean-squared atomic displacements perpendicular to the graphite planes, estimated from the Debye-Waller analysis of the intensity drops in (a). (c) Effective surface potential $V_{s}$ at the HOPG surface as a function of the applied fluence $F$. (d) Decay of the effective surface potential with fit to the driftdiffusion recombination model (see text). (e) Contraction of the interlayer spacing $\Delta d$ (left axis) and the corresponding increase in the effective surface potential (right axis) in the far-from equilibrium regime.

at $F=40 \mathrm{~mJ} / \mathrm{cm}^{2}$, which would correspond to an interplanar expansion of $6 \%$. Such a large peak shift cannot arise from structural changes alone.

We believe that the key effect of the optical excitation is the repopulation of electronic states, causing rebonding and redistribution of charge at the surface [18]. This charge redistribution gives rise to a Coulomb field near the graphite surface, which induces a collective shift of the electron diffraction pattern. We measure this Coulomb field here directly by modeling the 'refraction' effect commonly associated with the existence of a surface potential at the vacuum-material interface [18]. We deduce the effective surface potential $V_{s}$ as a function of the applied fluence and find it to increase linearly up to $F \approx 80 \mathrm{~mJ} / \mathrm{cm}^{2}$, as shown in Fig. 2(c). This linear trend indicates that the surface charging is mainly driven by the non-equilibrium diffusion of photogenerated carriers rather than multiphoton ionization. The decay of this transient surface potential resulting from the recombination of space charge follows a power-law decay with the exponent -1 , as shown in Fig. 2(d) and predicted by the drift-diffusion recombination model [18].

To examine the effect of optical excitations on the atomic structure at these elevated fluences, we monitored the interlayer separation following the photo-excitation by a time-resolved LDF analysis, after having accounted for the surface potential effects. In contrast to the thermal expansion at low fluences, we observed the interlayer separation to contract rapidly, as seen in Fig. 2(e). The 

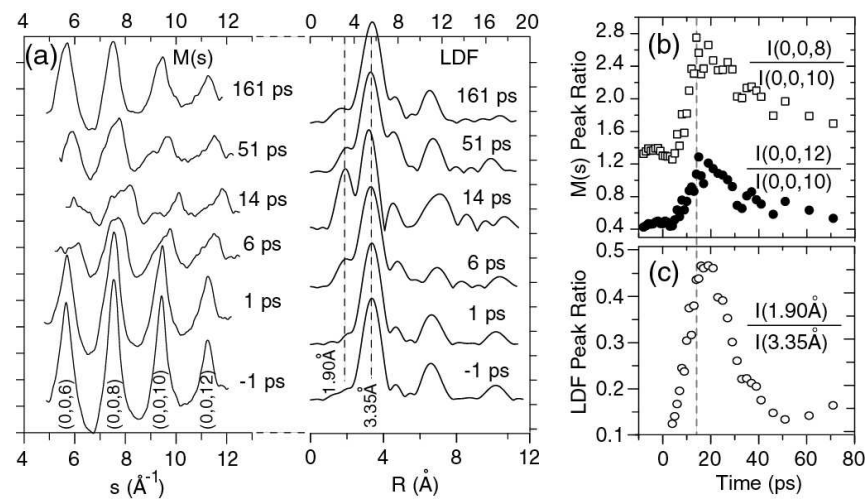

FIG. 3: Signature of $s p^{3}$-like bonding in HOPG. (a) Molecular interference pattern $M(s)$ and the corresponding LDF curves at selected time stances following strong photoexcitation. The transient peak at $1.9 \AA$ in the LDF indicates formation of an interlayer bond. (b) Ratio of peak intensities obtained from the diffraction patterns. The enhancement of the $(0,0,12)$ with respect to the $(0,0,10)$ peak cannot be explained by thermal vibrations and indicates a transient structure consistent with $s p^{3}$ bonding. (c) Ratio of peak intensities at $R=1.9 \AA$ and $3.35 \AA$ in the LDF.

change in the interlayer spacing $\Delta \mathrm{d}$ is found to correlate well with the rise and fall of $V_{s}$ at $F=77 \mathrm{~mJ} / \mathrm{cm}^{2}$, with a maximum value $V_{s} \approx 12 \mathrm{~V}$ corresponding to an interlayer contraction of $\approx 6 \%$. The potential rise $V_{s}$ sampled within the electron probe depth of $1 \mathrm{~nm}$ yields an internal field of $E \approx 1.2 \mathrm{~V} / \AA$, which causes Coulomb stress. Using this value of $E$ and $\epsilon_{r}=10$ for graphite, we estimate a maximum energy density of $U_{e}=\epsilon_{r} \epsilon_{0} E^{2} / 2=$ $0.2 \mathrm{eV} /$ atom, close to the $\approx 0.3 \mathrm{eV} /$ atom value based on DFT for the activation barrier in the graphite-todiamond transformation [3, 4 ].

To further elucidate the structural evolution, we examined closely the diffraction spectra. Figure 3(a) shows the time evolution of the molecular interference curves $M(s)$ and the corresponding LDF curves at $F=77 \mathrm{~mJ} / \mathrm{cm}^{2}$, just below the damage threshold. Starting at $\approx 6 \mathrm{ps}$, as $V_{s}$ rises beyond $4 \mathrm{~V}$, we observe the onset of peak shouldering and broadening in the $M(s)$ curve and appearance of a new peak at $R \approx 1.9 \AA$ in the LDF curve. Concurrent with this, we also observe a drastic change of relative intensities between the even and odd peaks in the $M(s)$ curves. In graphite with the interlayer spacing of $d_{0}=c_{0} / 2=3.35 \AA$, the even Bragg peaks $n=4,6$ are labeled $(0,0,8)$ and $(0,0,12)$, and the odd peaks $n=3,5$ are labeled $(0,0,6)$ and $(0,0,10)$, respectively. The observed higher intensity of the $(0,0,12)$ peak with respect to the $(0,0,10)$ peak in Fig. 3(b) cannot be explained by thermal vibrations and suggests a significant structural change. Consistent with the fact that only the even diffraction peaks should be present in the structure of diamond, and also with the observation of a well-defined interlayer bond at $R \approx 1.9 \AA$, we conclude that the tran- sient structure is consistent with $s p^{3}$ bonding. We find the transient $s p^{3}$-like structure, associated with the relative intensity of the $R \approx 1.9 \AA$ peak in the LDF curve, to reach a maximum fraction of $47 \%$ at $14 \mathrm{ps}$, as seen in Fig. 3 (c). Similar $s p^{2}-s p^{3}$ hybrid structures have also been identified in pressure-driven pathways of graphiteto-diamond transition [19]. By $45 \mathrm{ps}$, the structure recovers its $s p^{2}$ character, but remains hot even after $3 \mathrm{~ns}$ with an average value $\Delta \bar{u}^{2}=0.033 \AA^{2}$, corresponding to a lattice temperature $T \approx 1000 \mathrm{~K}$.

To clarify the origin of the structural changes, we studied the effect of laser pulses on the electronic structure and bonding in hexagonal graphite using $a b$ initio DFT calculations in the local density approximation (LDA). While much of the basic physics underlying photo-induced structural changes can be understood in the bulk structure, we used graphite slabs representing the surface for quantitative predictions. The total energy was determined using the ABINIT plane-wave code [20] with a 64 Ry energy cutoff, Troullier-Martins pseudopotentials, and the Ceperley-Alder form of the exchangecorrelation functional. The Brillouin zone of the 4 -atom bulk unit cell was sampled using a fine mesh of $24 \times 24 \times 12$ $k$-points that included the $K-H$ line at the Brillouin zone edge, close to the Fermi surface. This approach correctly reproduced the observed [16] in-layer bond length $d_{C C}=1.42 \AA$ and the interlayer spacing $d_{0}=3.34 \AA$ in the bulk system at $T=0$, shown schematically in Fig. 4(a). The electronic density of states of graphite near the Fermi level is shown in Fig. 4(b), and the total charge density is depicted in Fig. 4(c) in a plane normal to the layers. In the following, we will study the effect of two types of photo-induced electronic excitations on the structure.

First, we will consider the thermalization of the initial nonequilibrium population of electronic states in the laser-irradiated target over a sub-picosecond time scale 21] to that of a very hot electron gas [22]. The electronic temperature $\left(T_{e}\right)$ is probably lower than the limiting value $k_{B} T_{e} \lesssim h \nu=1.55 \mathrm{eV}$ and depends on the laser fluence. For the sake of illustration, we compare the Fermi-Dirac distribution in graphite at $k_{B} T_{e}=0$ and $k_{B} T_{e}=1.0 \mathrm{eV}$ in Fig. 4(b), indicating the electronic excitations in the $\pi-\pi^{*}$ manifold of the hot electron gas. Populating initially empty conduction states by valence electrons leads to a change in the total electron density $\Delta \rho(\mathbf{r})=\rho\left(\mathbf{r} ; k_{B} T_{e}\right)-\rho(\mathbf{r} ; 0)$, which is depicted in Fig. $4(\mathrm{~d})$ for $k_{B} T_{e}=1.0 \mathrm{eV}$.

As stipulated by DFT, changes in the charge density modify the force field in the system. Our results in Fig. 4(d) suggest an increased population of $\mathrm{C} 2 p_{z}$ orbitals that may hybridize to $p p \sigma$ bonding states connecting neighboring layers, increasing their attraction. The depopulation of in-layer bonding states, on the other hand, should cause an in-layer expansion. To obtain a quantitative estimate of photo-induced structural changes, we 

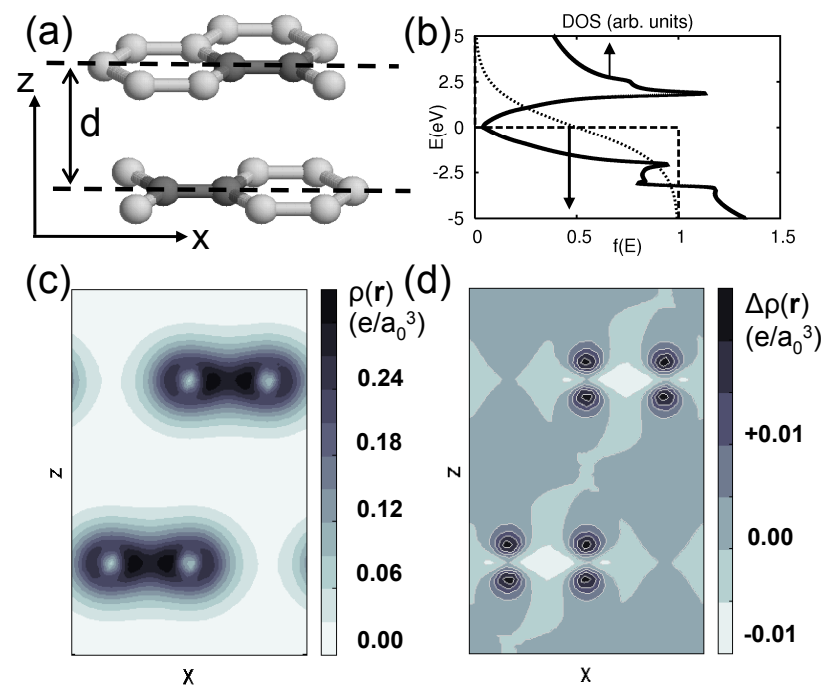

FIG. 4: (Color online) Electronic structure changes during photo-excitations in graphite. (a) Schematic view of the structure. (b) Electronic density of states (solid line) and the Fermi-Dirac distribution of graphite at $T=0$ (dashed line) and $k_{B} T=1.0 \mathrm{eV}$ (dotted line). (c) Total pseudo-charge density $\rho(\mathbf{r})$ and (d) change in the pseudo-charge density $\Delta \rho(\mathbf{r})$ due to an electronic temperature increase to $k_{B} T=1.0 \mathrm{eV}$. The plane used in (c) and (d) intersects the graphene layers along the dashed lines in (a).

performed a set of global structure optimization calculations of graphite with electrons subject to effective temperatures in the range $0 \mathrm{eV}<k_{B} T<1.55 \mathrm{eV}$. We used a stringent convergence criterion, requiring that all stress components lie below $5.0 \times 10^{-7} \mathrm{Ha} / \mathrm{a}_{0}^{3}$ and that no force exceeds $5.0 \times 10^{-5} \mathrm{Ha} / \mathrm{a}_{0}$. In bulk graphite, we indeed found that nonzero electronic temperature leads to a maximum interlayer contraction $\Delta d / d_{0}=-1 \%$ and inlayer expansion $\Delta r_{C C} / r_{C C}=+1 \%$ for $k_{B} T_{e} \approx 1.0 \mathrm{eV}$. In the slab geometry, we obtained a slightly larger interlayer contraction of up to $\Delta d / d_{0}=-1.5 \%$.

To further take into account the effect of Coulomb stress induced by the laser pulse, we represent the charge separation in the electronic ground state, corresponding to the observed internal field of $\approx 1.2 \mathrm{~V} / \AA$. To model this system, we immersed three-layer graphite slabs, separated by $30 \AA$, in a uniform electric field and adjusted its strength to reproduced the observed field value. With the polarized charge distribution frozen in, the external electric field was switched off. Then, the internal field was only due to the polarized charge distribution. The slab calculations were performed using the SIESTA code 23] with double-polarized triple- $\zeta$ local basis and a fine $24 \times 24 k$-point mesh. The charge density was obtained on a real-space grid with a mesh cutoff energy of $250 \mathrm{Ry}$. From total energy calculations at different interlayer separations, we found that charge density redistribution associated with the internal field may further reduce the interlayer separation by $2-3 \%$. Combined with the con- traction induced by the initial non-equilibrium electron heating, we thus can explain the observed contraction of the topmost interlayer separation by up to $\approx 5 \%$.

In conclusion, a nonthermal pathway of photoinduced structural changes in graphite has been observed through multidimensional crystallographic determination in space, time and energy. Beyond a threshold fluence, a transient $s p^{3}$-like structure with well-defined interlayer bonds emerges. The modified force field in the excited state and the Coulomb stress are the main forces driving this structural change.

This work was supported by Department of Energy under grant DE-FG02-06ER46309 (RKR, YM, and CYR), and by the National Science Foundation under NSFNSEC grant 425826 and NSF-NIRT grant ECS-0506309 (SB, TY and DT).

* Email: ruan@pa.msu.edu

$\dagger$ Email: tomanek@pa.msu.edu

[1] K. Nasu, ed., Photoinduced Phase Transitions (World Scientific, Hackensack, USA, 2004).

[2] T. Meguro, et al., Appl. Phys. Lett. 79, 3866 (2001).

[3] H. Nakayama and H. Katayama-Yoshida, J. Phys: Condens. Matter 15, R1077 (2003).

[4] S. Fahy, S. G. Louie, and M. L. Cohen, Phys. Rev. B 34, 1191 (1986).

[5] G. W. Yang and J. B. Wang, Appl. Phys. A: Mater. Sci. Process. 72, 475 (2001).

[6] S. Ashitkov, et al., JETP Lett. 75, 87 (2002).

[7] D. H. Reitze, H. Ahn, and M. C. Downer, Phys. Rev. B 45, 2677 (1992).

[8] T. Mishina, K. Nitta, and Y. Masumoto, Phys. Rev. B 62, 2908 (2000).

[9] K. Ishioka, M. Hase, M. Kitajima, and K. Ushida, Appl. Phys. Lett 78, 3965 (2001).

[10] M. Bargheer, N. Zhavoronkov, M. Woerner, and T. Elsaesser, ChemPhysChem 7, 783 (2006).

[11] A. H. Zewail, Ann. Rev. Phys. Chem. 57, 65 (2006).

[12] J. R. Dwyer, et al., Phil. Trans. R. Soc. A 364, 741 (2006).

[13] H. Park, et al., Solid State Commun. 136, 559 (2005).

[14] C.-Y. Ruan, Y. Murooka, R. K. Raman, and R. A. Murdick, Nano Lett. 7, 1290 (2007).

[15] A. Janzen, et al., Surf. Sci. 600, 4094 (2006).

[16] B. T. Kelly, Physics of Graphite (Applied Science, London, 1981).

[17] T. Kampfrath, et al., Phys. Rev. Lett 95, 187403 (2005).

[18] R. A. Murdick, R. K. Raman, Y. Murooka, and C.-Y. Ruan, Phys. Rev. B 77, 245329 (2008).

[19] W. L. Mao, et al., Science 302, 425 (2003).

[20] X. Gonze, et al., Comput. Mat. Sci. 25, 478 (2002).

[21] Y. Miyamoto, A. Rubio, and D. Tománek, Phys. Rev. Lett. 97, 126104 (2006).

[22] P. L. Silvestrelli, A. Alavi, M. Parrinello, and D. Frenkel, Phys. Rev. Lett. 77, 3149 (1996).

[23] J. M. Soler, et al., J. Phys.: Condens. Matter 14, 2745 (2002). 